\title{
Neuroendocrine tumor of thoracic spine: case report and literature review
}

\author{
Huiquan Gao ${ }^{1,2 \#}$, Yanzhen Wan ${ }^{3 \#}$, Hongxia $\mathrm{Ma}^{4}$, Tao Huang ${ }^{5}$, Wei Song ${ }^{6}$ \\ ${ }^{1}$ Graduate School, Shandong University of Traditional Chinese Medicine, Jinan, China; ${ }^{2}$ Department of Oncology, the 970 Hospital of PLA, Weihai, \\ China; ${ }^{3}$ Clinical Laboratory, Qingdao Women and Children's Hospital, Qingdao Women and Children's Hospital Affiliated to Qingdao University, \\ Qingdao, China; ${ }^{4}$ Shandong First Medical University \& Shandong Academy of Medical Sciences, Tai'an, China; ${ }^{5}$ Department of Radiology, the 970 \\ Hospital of PLA, Weihai, China; ${ }^{6}$ Department of Oncology, Shandong Provincial Hospital Affiliated to Shandong First Medical University, Jinan, \\ China \\ "These authors contributed equally to this work. \\ Correspondence to: Dr. Wei Song. Department of Oncology, Shandong Provincial Hospital Affiliated to Shandong First Medical University, 324 Jing \\ Wu Road, Jinan 250021, China. Email: tougao13406738582@126.com.
}

\begin{abstract}
Neuroendocrine tumors (NETs) generally arise from endocrine cells of gut and bronchi. Primary NETs of spine are extremely rare and have been described in cervical spine, lumbar spine, sacrum, and coccyx. So far, primary NETs in thoracic spine have not been reported yet. Here we described a 46-year-old Chinese woman with NET in thoracic spine. Neuroimaging revealed a mass behind the vertebral body of T11, abnormal changes in the adnexa and surrounding soft tissue and compression of the spinal cord. She received a total resection of the tumor and T11 corpectomy. Histopathological examination and immunohistochemical staining proved the tumor to be a rare spinal NET. PET-CT and other examinations ruled out the existence of tumors in any other site. So, she was diagnosed with NET of thoracic spine. The patient received postoperative etoposide and nedaplatin chemotherapy for four cycles, and she recovered well with no evidence of tumor recurrence or metastasis during six-month medical follow-up. Spine location of NETs should be first considered as a metastatic disease unless there is proof ruling out the possibility. Complete tumor resection is the most effective therapy in NETs of spine and should be considered in priority, and chemotherapy and radiotherapy should be considered on an individual basis.
\end{abstract}

Keywords: Thoracic spine; neuroendocrine tumor (NET); chemotherapy; case report

Submitted Jun 07, 2020. Accepted for publication Oct 26, 2020.

doi: $10.21037 /$ tcr-20-2279

View this article at: http://dx.doi.org/10.21037/tcr-20-2279

\section{Introduction}

Neuroendocrine tumors (NETs) are a heterogeneous group of epithelial neoplastic lesions (1). The incidence of NETs has been reported to be 1 in 100,000 (2). The digestive system and lungs are the most common sites of NETs (3). Primary NETs of spine are extremely rare and have been described in cervical spine (4), lumbar spine (2), sacrum and coccyx (5-7). No specific symptoms are observed in primary NETs of spine. Bone pain is by far the most common symptom. Immunohistochemical markers are crucial for a confirmatory diagnosis. And plasma levels of chromogranin and tyrosine kinase-2 have been proposed as specific biomarkers for NETs $(8,9)$. So far, primary NETs in thoracic spine have not been reported yet. Here we reported a case of NET in thoracic spine. We present the following article in accordance with the CARE reporting checklist (10) (available at http://dx.doi.org/10.21037/tcr-20-2279).

\section{Case presentation}

The patient was a 46-year-old Chinese woman of Han nationality. She was admitted to the Department of Spinal Surgery of Shandong Provincial Hospital Affiliated 

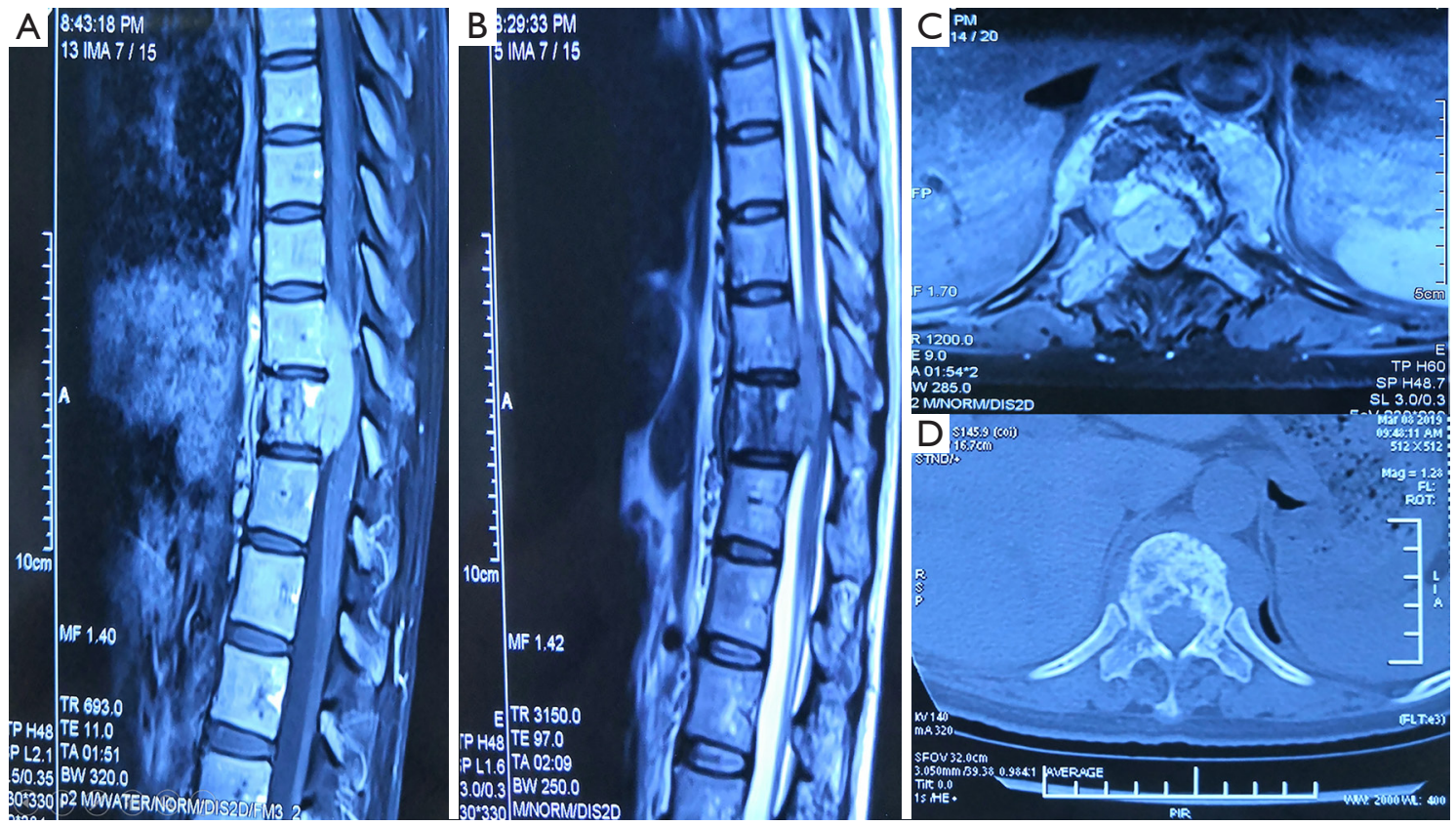

Figure 1 Preoperative magnetic resonance imaging and computed tomography of the thoracic spine. (A,B,C) Magnetic resonance imaging revealed a mass behind the vertebral body of T11 bulging into the spinal canal, spinal stenosis, indentation of the spinal cord, and enhancing homogeneity with contrast. The mass demonstrated isointense signal on T1-weighted images and mixed signal on T2-weighted images. (D) Computed tomography scan showed discontinuity of bone cortex of T11.

to Shandong First Medical University in March 2019, complaining of loss of motor and sensory function in lower extremities and back pain for six months. A pinprick sensation test showed bilateral loss of motor and sensory function in lower extremities. She received symptomatic treatments in the local hospital, with poor therapeutic effect. The patient was healthy before, with no family history of tumors. She denied any recent trauma or injury. Magnetic resonance imaging (MRI) of the spine (Figure $1 A, B, C$ ) revealed a mass behind the vertebral body of T11 bulging into the spinal canal, spinal stenosis, indentation of the spinal cord, and enhancing homogeneity with contrast. The mass demonstrated isointense signal on T1-weighted images and mixed signal on T2-weighted images. Computed tomography (CT) imaging showed discontinuity of bone cortex of T11 (Figure 1D). Owing to the limited condition of our hospital, the patient didn't receive ${ }^{68} \mathrm{Ga}$-PET/CT examination. Under general anesthesia, a total resection of the tumor, T11 corpectomy and bone grafting with instrumented fixation in a standard fixation surgery were performed for her. The postoperative pathological tissue of the patient was detected and analyzed by the Department of Pathology of Shandong Provincial
Hospital and Qianfoshan Hospital of Shandong Province. Histopathological examination indicated that the tissue was low-grade NET (Figure 2). Immunohistochemical staining revealed that the tumor cells were positive for chromogranin A, synaptophysin, CK8/18, CAM5.2, cytokeratin, and Vimentin (focal) and negative for TTF-1, LCA, CD56 and S-100. The Ki-67 labeling index was 15\%, indicating that the tumor was G2 grade. Postoperative CT and PETCT revealed complete excision of the lesion (Figure 3) and no other lesions were observed. So, the patient was finally diagnosed with NET (G2) of thoracic spine. She recovered well from the operation and then received postoperative etoposide and nedaplatin chemotherapy for four cycles in the Department of Oncology. The timeline of the patient was listed in Table 1. By now, the patient has received 1-year follow-up examination. Clinical symptoms were markedly improved. Results of routine blood examination, liver and kidney function and tumor marker levels were basically normal, and CT scan of chest and abdomen revealed no evidence of tumor recurrence or metastasis.

All procedures performed in studies involving human participants were in accordance with the ethical standards of the institutional and/or national research committee(s) and 
Table 1 Timeline of the patient

\begin{tabular}{|c|c|c|}
\hline Date & Preoperative examination and treatment & Postoperative examination and treatment \\
\hline March 18 & - & $\begin{array}{l}\text { Histopathological examination and immunohistochemical } \\
\text { staining proved the mass to be a low-grade neuroendocrine } \\
\text { tumor (G2) }\end{array}$ \\
\hline April 24 to July 29 & - & Four cycles of etoposide and nedaplatin chemotherapy \\
\hline
\end{tabular}

PET-CT, positron emission tomography-computed tomography.

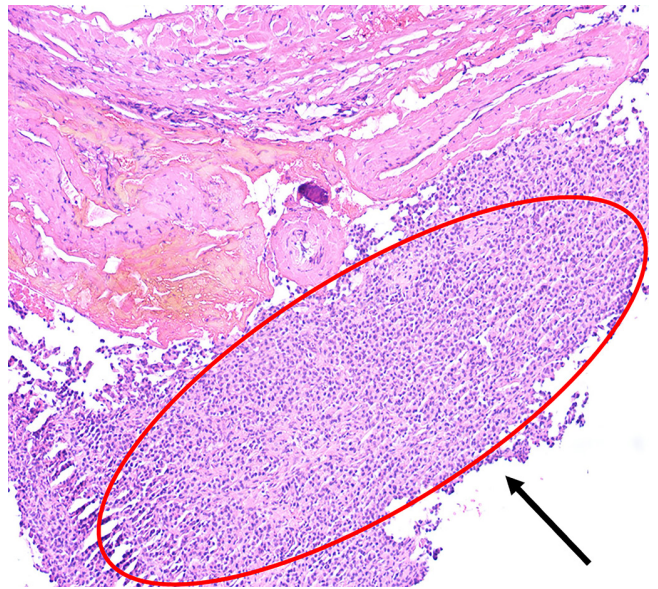

Figure 2 Histopathological examination showed plump small round cells (HE staining, $\times 40)$.

with the Helsinki Declaration (as revised in 2013). Written informed consent was obtained from the patient.

\section{Discussion}

NETs are heterogeneous neoplasms arising from secretory cells of the diffuse neuroendocrine system, which predominate in gastroenteropancreatic tract and bronchopulmonary tree (11). Spine NETs are extremely rare and are usually secondary outcomes of metastatic diseases (12-14). Bone metastasis of NETs was often accompanied by other distant metastasis like liver metastasis. 53-87\% of patients with bone metastasis would be complicated by liver metastasis (13). The first diagnostic hypothesis for

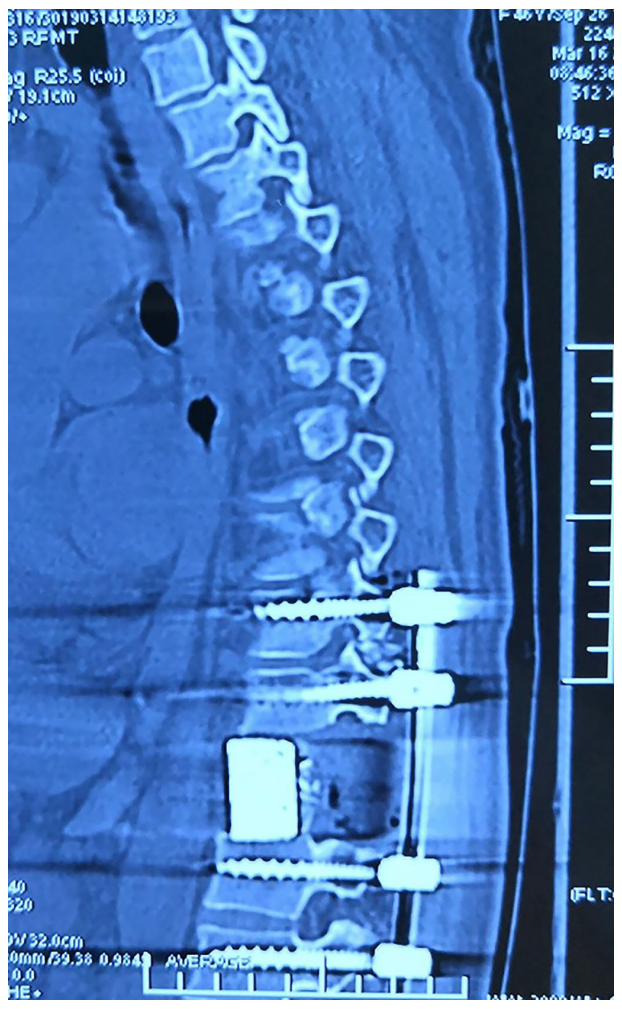

Figure 3 Postoperative computed tomography revealed complete excision of the lesion and T11 corpectomy. Bone grafting with instrumented fixation was performed.

our patient is just the metastasis from an unknown primary NET, but complete postoperative examination and followup failed to reveal any other NET lesions elsewhere in the body. 
NETs usually remain clinically silent for a long period and are often discovered in the context of nerve or organ compression (5). Symptoms are mainly related to the site of tumor and whether there is secretory function. In the case of spinal lesions, mass effects can include local or radicular pain, radiculopathy, and myelopathy if spinal cord is involved. Our patient presented with back pain and neurological symptoms, such as paresthesia and serious weakness in bilateral lower extremities. Some NETs can secrete vasoactive peptides and amines, such as serotonin and tachykinins, resulting in carcinoid syndrome. The common symptoms of carcinoid syndrome include diarrhea, flushing, bronchospasm, and telangiectasia (15). There were no signs of hormonal imbalance in our patient, so the tumor was supposed to be a spine NET without secretory function.

It is difficult to diagnose primary NETs of spine preoperatively just by imaging features. It needs to be differentiated with bone metastasis of common cancers such as lung cancer and breast cancer, and also as an extradural thoracic spinal mass, the differential diagnosis for NET of thoracic spine includes paraganglioma, meningioma, hematoma, neurofibroma, metastasis, abscess-phlegmon, multiple myeloma, lymphoma, paraganglioma, angiolipoma, and so on $(5,16)$. Imaging examination plays an important role in the detection and diagnosis of NETs. CT scan is sensitive in the diagnosis of bone cortex destruction. MRI can better identify the condition of lesion and surrounding soft tissue and nerve involvement. And spinal MRI is important for the detection of compression of spinal cord and spine stability. Preoperative T1-weighted MRI of the lumbar spine that reveals isointense and contrast-enhanced masses are more likely to result in a definite diagnosis than CT (8). Emission computed tomography (ECT) can be used to screen bone lesions in other sites. The ability to detect skeletal NETs has dramatically increased since the introduction of ${ }^{68} \mathrm{Ga}-\mathrm{PET} / \mathrm{CT}$. It is reported that the diagnostic accuracy of ${ }^{68} \mathrm{Ga}-\mathrm{PET} / \mathrm{CT}$ in identifying NET bone lesions is between $80 \%$ and $100 \%$. Compared to contrast-enhanced CT and MRI, ${ }^{68} \mathrm{Ga}-\mathrm{PET} / \mathrm{CT}$ may provide a $20-25 \%$ yield in terms of diagnostic ability to detect bone lesions (17). In our case, CT, MRI, ECT and PET-CT have been used in combination to precisely present this lesion in the thoracic spine and rule out other lesions.

The confirmative diagnosis of NETs is based on histopathological examination and immunohistochemical analysis. The histopathological features include nests and trabeculae of cells exhibiting round or oval nuclei and rare mitoses, which are characteristics of a NET (4). Immunohistochemical markers are crucial for a confirmatory diagnosis of NETs, and the tumor is usually observed to be positive for chromogranin A and synaptophysin (4). The 2010 WHO classification distinguishes neuroendocrine neoplasms (NENs) in three groups: G1 (Ki-67 $\leq 2 \%)$, G2 (Ki-67 3-20\%) and G3 (Ki$67>20 \%$ ), according to Ki-67 value. G1 and G2 grades are NETs, and G3 is neuroendocrine carcinoma (NEC) (14). The pathological tissue of our patient was detected and analyzed by the Department of Pathology of two tertiary hospitals, confirming the diagnosis of NET belonging to G2 with Ki-67 of $15 \%$.

Total resection of the tumor is the most effective treatment for patients with NETs, especially for those with localized tumors. Radiotherapy is the best auxiliary choice for patients with spinal cord metastasis of NETs (18). Some patients may also benefit from chemotherapy, but there is limited evidence for the therapeutic effect of chemotherapy and radiotherapy in the management of primary NETs of spine $(8,19)$. A complete tumor resection was performed in our patient, and 4 cycles of chemotherapy were given after surgery for further disease control. Our patient didn't receive any radiotherapy for some personal reason. However, localized radiotherapy is one of the most important treatment strategies for lesion sites where it is impossible to perform expanded resection. With advances in gene monitoring and targeted treatments, individualized treatment according to individual profile is expected to become a potential and promising strategy.

For localized NETs, relative 5-year survival rate is approaching $93 \%$, not significantly different among the different primary sites (20). Regular re-examination is necessary to facilitate early detection of tumor recurrence, and discovery of small lesions not found at the beginning of diagnosis. Our patient has been followed up for one year. No recurrence in the thoracic spine and no lesions in other parts of the body were found, indicating that she might have suffered from a primary NET of thoracic spine. However, long term re-examination is still needed to rule out the possibility of metastasis from other sites.

\section{Conclusions}

Thoracic spine NET is a rare entity and is difficult to be diagnosed preoperatively. Confirmatory diagnosis depends on histopathological examination and immunohistochemical 
analysis. Spine location of NETs should be first considered as a metastatic disease unless there is proof ruling out the possibility. Complete tumor resection is the most effective therapy in NETs of spine and should be considered in priority, and chemotherapy and radiotherapy should be considered on an individual basis.

\section{Acknowledgments}

Funding: This study was supported by grants from the Shandong Province Science and Technology Development Plan of Traditional Chinese Medicine (No. 2019-0313).

\section{Footnote}

Reporting Checklist: The authors have completed the CARE reporting checklist. Available at http://dx.doi.org/10.21037/ tcr-20-2279

Conflicts of Interest: All authors have completed the ICMJE uniform disclosure form (available at http://dx.doi. org/10.21037/tcr-20-2279). The authors have no conflicts of interest to declare.

Ethical Statement: The authors are accountable for all aspects of the work in ensuring that questions related to the accuracy or integrity of any part of the work are appropriately investigated and resolved. All procedures performed in studies involving human participants were in accordance with the ethical standards of the institutional and/or national research committee(s) and with the Helsinki Declaration (as revised in 2013). Written informed consent was obtained from the patient. All patient identifiers have been removed.

Open Access Statement: This is an Open Access article distributed in accordance with the Creative Commons Attribution-NonCommercial-NoDerivs 4.0 International License (CC BY-NC-ND 4.0), which permits the noncommercial replication and distribution of the article with the strict proviso that no changes or edits are made and the original work is properly cited (including links to both the formal publication through the relevant DOI and the license). See: https://creativecommons.org/licenses/by-nc-nd/4.0/.

\section{References}

1. Perren A, Couvelard A, Scoazec JY, et al. ENETS consensus guidelines for the standards of care in neuroendocrine tumors: Pathology: Diagnosis and prognostic stratification. Neuroendocrinology 2017;105:196-200.

2. Kumar S, Maheshwari V, Mukherjee A, et al. Primary neuroendocrine tumor of the lumbar spine: Rare tumor mimicking nerve sheath tumor of the spine. Asian J Neurosurg 2019;14:894-96.

3. Dasari A, Shen C, Halperin D, et al. Trends in the incidence, prevalence, and survival outcomes in patients with neuroendocrine tumors in the united states. JAMA Oncol 2017;3:1335-42.

4. Narayanan M, Serban D, Tender GC. Primary cervical spine carcinoid tumor in a woman with arm paresthesias and weakness: A case report. J Med Case Rep 2013;7:214.

5. Dujardin F, Beaussart P, de Muret A, et al. Primary neuroendocrine tumor of the sacrum: Case report and review of the literature. Skeletal Radiol 2009;38:819-23.

6. Krasin E, Nirkin A, Issakov J, et al. Carcinoid tumor of the coccyx: Case report and review of the literature. Spine (Phila Pa 1976) 2001;26:2165-67.

7. Schnee CL, Hurst RW, Curtis MT, et al. Carcinoid tumor of the sacrum: Case report. Neurosurgery 1994;35:1163-7.

8. Zhang XF, Zhang Y, Yan X, et al. Primary carcinoid tumor of medulla spinalis: Case report and review of the literature. Eur J Med Res 2014;19:71.

9. Melen-Mucha G, Niedziela A, Mucha S, et al. Elevated peripheral blood plasma concentrations of tie- 2 and angiopoietin 2 in patients with neuroendocrine tumors. Int J Mol Sci 2012;13:1444-60.

10. Gagnier JJ, Kienle G, Altman DG, et al. The CARE guidelines: Consensus-based clinical case reporting guideline development. J Med Case Rep 2013;7:223 .

11. Cives M, Strosberg JR. Gastroenteropancreatic neuroendocrine tumors. CA Cancer J Clin 2018;68:471-87.

12. Hori T, Yasuda T, Suzuki K, et al. Skeletal metastasis of carcinoid tumors: Two case reports and review of the literature. Oncol Lett 2012;3:1105-8.

13. Van Loon K, Zhang L, Keiser J, et al. Bone metastases and skeletal-related events from neuroendocrine tumors. Endocr Connect 2015;4:9-17.

14. Pavel M, Baudin E, Couvelard A, et al. ENETS Consensus Guidelines for the management of patients with liver and other distant metastases from neuroendocrine neoplasms of foregut, midgut, hindgut, and unknown primary. Neuroendocrinology 2012;95:157-76.

15. Li C, Huang J, Yang X, et al. A primary neuroendocrine 
tumor of the left ventricle presenting with diarrhea-an unusual experience and literature review. Diagn Pathol 2020;15:32.

16. Reddy A, Moron F. Primary extradural paraganglioma of the thoracic spine: A case report. J Radiol Case Rep 2017;11:1-7.

17. Cives M, Pelle' E, Rinzivillo M, et al. Bone metastases in neuroendocrine tumors: Molecular pathogenesis and implications in clinical practice. Neuroendocrinology 2021;111:207-16.

Cite this article as: Gao H, Wan Y, Ma H, Huang T, Song W. Neuroendocrine tumor of thoracic spine: case report and literature review. Transl Cancer Res 2021;10(2):1177-1182. doi: 10.21037/tcr-20-2279
18. Li D, Brennan JW, Buckland M, et al. Bronchogenic carcinoid metastasis to the intramedullary spinal cord. J Clin Neurosci 2010;17:1196-98.

19. Lal A, Chen H. Treatment of advanced carcinoid tumors. Curr Opin Oncol 2006;18:9-15.

20. Quaedvlieg PF, Visser O, Lamers CB, et al. Epidemiology and survival in patients with carcinoid disease in the Netherlands. An epidemiological study with 2391 patients. Ann Oncol 2001;12:1295-300. 